\title{
ON THE GENERALIZATION OF A DIRECT METHOD OF THE CALCULUS OF VARIATIONS ${ }^{1}$
}

\author{
BY GEORGE J. MINTY
}

Communicated by F. E. Browder, January 23, 1967

1. Introduction. Consider a topological linear space $V$, with adjoint space $V^{*}$, and a function $f$ defined on a domain in $V$ and taking on values in $V^{*}$; one wishes to prove the existence of a solution of the equation $f(x)=0$. A "standard" method is to show that $f$ is the Fréchet differential of a convex, real-valued functional $\phi$ and use the (virtually automatic) lower-semicontinuity of $\phi$ to show that $\phi$ has a minimum on some compact set in $V$ (which is usually taken with a "weak" topology). With appropriate asymptotic conditions on $\phi$, the compact set can be taken very large so that the minimum occurs at an interior point $x$, which then satisfies the equation. In this problem and a class of related problems, it has been found that the essential property of $f$ is that it is monotone in the sense: for all $x_{1}, x_{2}$, we have $\left\langle x_{1}-x_{2}, f\left(x_{1}\right)-f\left(x_{2}\right)\right\rangle \geqq 0$, and the existence of the scalar $\phi$ can often be dispensed with. (See [2] for a discussion of some of the limitations of the "monotonicity" method as opposed to the variational method.)

The "monotonicity" method, as presently constituted, is pretty much limited to Banach spaces, usually reflexive, and some aspects of the theory have been developed only in Hilbert space. Moreover, there are difficulties corresponding to the cases (in the variational method): $\left(1^{\circ}\right) \phi$ is not Fréchet-differentiable, but has a multiplevalued subgradient (see [14], [1]); $\left(2^{\circ}\right) \phi$ is only defined on a closed convex subset $C$ of $V$, or it is desired to minimize $\phi$ only over such a set; and $\left(3^{\circ}\right) \phi(x)$ does not "become infinite at infinite $x$ " but is at least "nonincreasing near infinity."

These difficulties have been overcome in various combinations, but usually with strong restrictions on the space $V$. The writer and F. E. Browder (see, e.g. [11]) treated $\left(3^{\circ}\right)$; the writer introduced the concept of "maximal monotone set" to deal with $\left(1^{\circ}\right)$, and treated $\left(1^{\circ}\right),\left(2^{\circ}\right)$, and $\left(3^{\circ}\right)$ simultaneously (see [13]) but for a Hilbert space $V$ and a closed linear subspace $C$. Recently Stampacchia and Lions [8], [9], [15] have seen how to deal with $\left(2^{\circ}\right)$ for general $C$, but for linear $f$ in a Hilbert space $V$. Lescarret [7] and Browder [2], [3] extended this latter work to nonlinear $f$ in a reflexive Banach space $V$.

1 This research was conducted with the support of an Alfred P. Sloan fellowship and of National Science Foundation grant GP-5229. 
The success of all these researches suggested to the writer that some simple principle might exist which has been overlooked in earlier research, and which would permit the redevelopment of the theory on a better foundation. We express, in Theorem 2 below, what appears to be such a principle. With its aid, we give a simple redevelopment of much of the existing theory in a very wide class of spaces (apparently wider than Banach spaces); the development parallels the variational method in that it works first with a compact set, and considers noncompact sets afterward. In addition, it makes substantial contributions to the treatment of $\left(1^{\circ}\right)$ above, and handles $\left(2^{\circ}\right)$ without difficulty. In the present paper, we do not succeed so well with $\left(3^{\circ}\right)$ in that we postulate $\langle x, f(x)\rangle>0$ near infinity, rather than the weaker $\langle x, f(x)\rangle \geqq 0$, to treat closed convex noncompact $C$; however, the stronger condition suffices for most needs of analysis. We make no attempt to treat the solution of $f(x, x)=0$, with $f$ monotone in first argument and completely continuous in second argument, leaving this for future development.

2. A theorem in the theory of linear inequalities. Let $V$ and $V^{1}$ be vector spaces over the reals, and let $\langle$,$\rangle be a bilinear form$ mapping $V \times V^{1}$ in to $R$. (Example: if $V$ is a complex linear space and $V^{1}$ a space of linear functionals on $V$, then $\langle x, y\rangle=\operatorname{Re} y(x)$ is such a form.) In the sequel, the word "monotone" will always be meant with reference to $\langle$,$\rangle .$

THEOREM 1. Let $\left\{\left(x_{i}, y_{i}\right): i=1, \cdots, m\right\}$ be a sequence of pairs in $V \times V^{1}$ such that, for $i, j=1, \cdots, m$, we have $\left\langle x_{i}-x_{j}, y_{i}-y_{j}\right\rangle \geqq 0$, i.e. a monotone sequence. Then there exists a vector $x$ such that for $i=1, \cdots, m$, we have $\left\langle x_{i}-x, y_{i}\right\rangle \geqq 0$.

This is a known theorem [10], [6], [4] which is inadequate because it does not give enough information on the location of $x$. (See [12] for an attempt to apply Theorem 1 to analysis-problems.) We remark, in passing, that if $\left\{\left(x_{i}, y_{i}\right)\right\}$ is contained in the subgradient of a convex functional, then $x$ can be chosen as one of the $x_{i}$, namely one which makes $\phi\left(x_{i}\right)$ a minimum. One can define a relation $\succ$ on $\{1, \cdots, m\}$ by $i \succ j$ provided $\left\langle x_{i}-x_{j}, y_{i}\right\rangle<0$, and then the "cyclic monotonicity" condition of Rockafellar [14] and a little bit of graph theory prove this fact. ( $\succ$ has no directed circuits.)

Theorem 2. Same as Theorem 1, but add: "... and $x$ contained in $K\left(x_{1}, \cdots, x_{m}\right)$, the convex hull of $\left\{x_{1}, \cdots, x_{m}\right\}$."

Proof. Recall the Fundamental Theorem of Game Theory: for 
any matrix of reals $\left[a_{i j}\right]$, there exist probability-vectors $\lambda^{0}$ and $\mu^{0}$ such that

$$
\begin{aligned}
\sum_{i, j} \lambda_{i}^{0} a_{i j} \mu_{j}^{0} & =\max _{\lambda} \sum_{i, j} \lambda_{i} a_{i j} \mu_{j}^{0}=\min _{\mu} \sum_{i, j} \lambda_{i}^{0} a_{i j} \mu_{j} \\
& =\max _{\lambda} \min _{\mu} \sum_{i, j} \lambda_{i} a_{i j} \mu_{j}=\min _{\mu} \max _{\lambda} \sum_{i, j} \lambda_{i} a_{i j} \mu_{j} .
\end{aligned}
$$

Now, if $\left[a_{i j}\right]$ is square and antisymmetric, the equality of the last two expressions shows that this number (the "value of the game") is zero. (One interchanges the dummy symbols $\lambda$ and $\mu$.) In the third of these five expressions, put $\mu$ equal a Kronecker-delta to see that for any antisymmetric matrix of reals $\left[a_{i j}\right]$, there exists a probability-vector $\lambda^{0}$ such that $\sum_{i} \lambda_{i}^{0} a_{i j} \geqq 0$ for all $j$. The same statement is true $a$ fortior $i$ for any $\left[a_{i j}\right]$ whose symmetric part has all nonnegative entries (by applying the above principle to the antisymmetric part). Let $a_{i j}=\left\langle x_{j}-x_{i}, y_{j}\right\rangle$, which has nonnegative symmetric part by the "monotonicity" hypothesis, to see $\left\langle x_{j}-\sum_{i} \lambda_{i}^{0} x_{i}, y_{j}\right\rangle \geqq 0$; Theorem 2 is proved.

There is very little "geometric intuition" to this theorem, which tends to explain why it went so long unseen. It is, however, suggested by the fact that the "monotonicity method" for analysis-problems (see [2], [3]) was developed over a convex set $C$; compactness is not sufficient alone, as in the variational method.

3. Applications. We now give our "main theorem":

THEOREM 3. Let $V, V^{1}$ be vector spaces over the reals, and let $\langle$, be a bilinear form on $V \times V^{1}$ into the reals. Let $V$ be topologized with any topology in which all of $\langle\cdot, y\rangle$ are continuous, and let $C$ be a compact convex set in $V$. Let $\left\{\left(x_{\alpha}, y_{\alpha}\right): \alpha \in A\right.$, an index set $\}$ be a monotone subset of $C \times V^{1}$ (for example, the graph of a monotone function with domain contained in $C)$. Then there exists $x \in C$ such that, for all $\alpha \in A$, we have $\left\langle x_{\alpha}-x, y_{\alpha}\right\rangle \geqq 0$.

Proof. The sets $C \cap\left\{x:\left\langle x_{\alpha}, y\right\rangle-\langle x, y\rangle \geqq 0\right\}$ have nonempty intersection when taken finitely many at a time, by Theorem 2 ; they are closed sets, by the continuity of $\langle\cdot, y\rangle$. The "finite intersection property" of compactness gives the conclusion.

Notice that it is irrelevant whether $V$ is locally-convex or a Hausdorff space, or even whether the vector-space operations are continuous, or the topology is invariant under the vector-space operations.

We now have to demonstrate that noncompact $C$ can be handled. 
Let us say that $V$ (taken with a topology) is "regular" provided (a) For any compact convex set $C$ and any point $x$, the closed convex hull of $C \cup\{x\}$ is compact, and (b) if a compact set $C$ contains one endpoint of a line-segment but omits the other, then it omits some third point of the line-segment. (Note (b) holds in any "topological vector space," since a one-dimensional topological vector space has the topology of the reals.)

Theorem 4. Assume $V, V^{1},\langle$,$\rangle and the topology on V$ as in Theorem 3; in addition, assume that $V$ is regular. Let $C$ be a closed convex subset of $V$, and let $f: C \rightarrow V^{1}$ be a function whose graph is monotone, i.e. a monotone function. Suppose $0 \in C$, and that there exists a compact set $D \subset C$ such that, for $x \notin D$ but $x \in C$, we have $\langle x, f(x)\rangle>0$. Then there exists a point $x \in D$ such that, for all $x^{\prime} \in C$, we have $\left\langle x^{\prime}-x, f\left(x^{\prime}\right)\right\rangle \geqq 0$.

Proof. For $x^{\prime} \in C$, consider the set $D \cap\left\{x:\left\langle x^{\prime}-x, f\left(x^{\prime}\right)\right\rangle \geqq 0\right\}$. It suffices to show these sets have nonempty intersection when taken finitely many at a time, corresponding to $x_{1}^{\prime}, \cdots, x_{m}^{\prime}$. Let $D^{\prime}$ be the closed convex hull of the union of $D$ with these points, and note $D^{\prime}$ is compact (since $V$ is regular). Apply Theorem 3 with $D^{\prime}$ in place of $C$, obtaining $x$ such that for all $x^{\prime} \in D^{\prime},\left\langle x^{\prime}-x, f\left(x^{\prime}\right)\right\rangle \geqq 0$, We now show $x \in D$. Suppose not. Since obviously $0 \in D$, and $V$ is regular, there exists a real $t$, with $0<t<1$, such that $t x \in D$; since $D^{\prime}$ is convex, $t x \in D^{\prime}$. Now, $\langle t x-x, f(t x)\rangle \geqq 0$; multiplying by $(t-1)^{-1} t$, which is negative, we see $\langle t x, f(t x)\rangle \leqq 0$, contradicting $t x \in D$.

Notice that Theorem 4 is easily modified to cover the case of multiple-valued $f$.

In order to see the connection between Theorems 3 and 4 and earlier work on "monotonicity methods" and applications, it is necessary to make an interpretation of the vector $x$. If the set $\left\{\left(x_{\alpha}, y_{\alpha}\right)\right\}$ (resp. the graph of $f$ ) is a maximal monotone set, then the interpretation is simple: the point $(x, 0)$ of $V \times V^{1}$ is contained in the set; in the "function" case, this means $f(x)=0$. See [14] concerning the maximal monotonicity of the subgradient of a convex functional, and [7] for interesting maximal monotone sets in Hilbert space. Another way of seeing the "meaning" of $x$ is provided by the following lemma:

Lemma 1. Let $V, V^{1}$, and $\langle$,$\rangle be as before, let V^{1}$ be topologized so all of $\langle x, \cdot\rangle$ are continuous, let $C$ be a convex set in $V$ (which need not be closed or compact) and let $f: C \rightarrow V^{1}$ be monotone, single-valued, and hemicontinuous in the sense of Browder (i.e., the restriction of $f$ to any line-segment in $C$, taken with its "natural" topology, is continuous). 
Then the sets $S=\left\{x: x \in C\right.$, and for all $\left.x^{\prime} \in C,\left\langle x^{\prime}-x, f\left(x^{\prime}\right)\right\rangle \geqq 0\right\}$ and $T=\left\{x: x \in C\right.$, and for all $\left.x^{\prime} \in C,\left\langle x^{\prime}-x, f(x)\right\rangle \geqq 0\right\}$ coincide.

Proof. (Both the Lemma and its proof are essentially due to Browder-it is virtually the same as Lemma 1 of [3] except for context.) The inclusion $T \subset S$ follows from the monotonicity of $f$; the other inclusion is obtained from $x, x^{\prime} \in C, 0<t \leqq 1$, by noting $x+t\left(x^{\prime}-x\right) \in C$ and taking the limit as $t \rightarrow 0$ in the inequality $t^{-1}\left\langle x+t\left(x^{\prime}-x\right)-x\right.$, $\left.f\left(x+t\left(x^{\prime}-x\right)\right)\right\rangle \geqq 0$.

(It is extremely important, for applications, that $f$ need not be continuous in the topology of $V$ corresponding to the compactness in Theorems 3 and 4. For example, in Hilbert space, this enables us to work with a weakly compact set and an $f$ which is continuous in the norm topology but not the weak topology.)

Thus, under the hypotheses of Theorem 4 (or Theorem 3) and Lemma 1 taken together, we have an existence theorem for a solution of the "variational inequalities of Stampacchia-Lions type" $\left\langle x^{\prime}-x, f(x)\right\rangle \geqq 0$; note these are nonlinear inequalities. The Lemma gives us the further information that the set of solutions is a closed convex set, since $S$ is obviously such a set. We note, as Stampacchia and Lions did, that if $C$ is a closed linear subspace of Hilbert space $V$, then the inequalities imply $f(x) \in C^{\perp}$, and if $C$ is all of $V$, they imply $f(x)=0$; further development of this idea is left to the reader.

4. Another convexity theorem. The writer believes that when this theory is put in "final form," the notion of "maximal monotone set" will be a central one, with "hemicontinuity" relegated to the secondary rôle of a tool for proving maximality. We therefore give another theorem which seems to be a relative of Lemma 1 above, and would be useful in that context. A set is called "antimonotone" if it satisfies the definition of "monotone," but with the inequality reversed.

ThEOREM 5. Let $V$ and $V^{1}$ be vector spaces over the reals, and $\langle$, be a bilinear form as before. (No topologies are assumed on $V$ and $V^{1}$,) Let $E$ and $F$ be maximal monotone and antimonotone sets, respectively, in $V \times V^{1}$, and let $S$ be the intersection, with $\left(x_{1}, y_{1}\right)$ running through $E$ and $\left(x_{2}, y_{2}\right)$ through $F$, of the sets $\left\{(x, y):\left\langle x_{1}-x, y_{1}-y\right\rangle-\left\langle x_{2}-x, y_{2}-y\right\rangle \geqq 0\right\}$. Then $S$ coincides with $E \cap F$.

Proof. It is trivial to show $(E \cap F) \subset S$. Consider $(x, y) \in S$, and suppose this point is not in $F$. By maximality of $F$, we obtain $\left(x_{2}, y_{2}\right)$ $\in F$ with

$$
\left\langle x_{2}-x, \quad y_{2}-y\right\rangle>0 .
$$


Thus for all $\left(x_{1}, y_{1}\right) \in E$, we have $\left\langle x_{1}-x, y_{1}-y\right\rangle$ strictly positive; but then by the maximality of $E,(x, y)$ must be in $E$, and hence $\langle x-x, y-y\rangle$ is strictly positive-a contradiction. The same argument, with some signs changed, shows $S \subset E$.

This theorem asserts, among other things, that $E \cap F$ is a convex set. As a sample application, consider the "Nonlinear Hammerstein Integral Equation" $x+K f x=0$ in an abstract Hilbert space, and suppose the graphs of $K$ and $f$ are maximal monotone sets. (The existence theorem for a solution is considered in [5].) Observe that the sets $\{(x, y): y=f(x)\}$ and $\{(x, y): x=-K y\}$ are maximal monotone and antimonotone sets, respectively, and that the set of solutions of the equation is a projection of this set; hence the solution set is convex.

Added in proof. A variant on Theorem 2 which matches Theorem 5 is as follows:

Theorem 6. Let $\left\{\left(x_{i}^{\prime}, y_{i}^{\prime}\right)\right\}$ and $\left\{\left(x_{j}^{\prime \prime}, y_{j}^{\prime \prime}\right)\right\}$ be finite monotone and antimonotone sets, respectively. Then there exists

$$
(x, y) \in K\left[\left(x_{i}^{\prime}+x_{j}^{\prime \prime}\right) / 2,\left(y_{i}^{\prime}+y_{j}^{\prime \prime}\right) / 2\right]
$$

such that for all $i, j$ we have $\left\langle x_{i}^{\prime}-x, y_{i}^{\prime}-y\right\rangle-\left\langle x_{j}^{\prime \prime}-x, y_{j}^{\prime \prime}-y\right\rangle \geqq 0$.

Proof. Let

$$
\begin{aligned}
a_{i j k l} & =\left\langle x_{i}^{\prime}-\left(x_{k}^{\prime}+x_{l}^{\prime \prime}\right) / 2, y_{i}^{\prime}-\left(y_{k}^{\prime}+\left(y_{k}^{\prime}+y_{l}^{\prime \prime}\right) / 2\right\rangle\right. \\
& -\left\langle x_{j}^{\prime \prime}-\left(x_{k}^{\prime}+x_{l}^{\prime}\right) / 2, y_{j}^{\prime \prime}-\left(y_{k}^{\prime}+y_{l}^{\prime \prime}\right) / 2\right\rangle .
\end{aligned}
$$

A routine but laborious calculation shows that $a_{i j k l}+a_{k l i j} \geqq 0$; the rest of the proof is like that of Theorem 2.

L. Nirenberg has observed that the methods of Hartman and Stampacchia ([16, Theorem 1.1]) are applicable to problems of the type considered here, and F. E. Browder [17], [18] has developed further the methods of the present paper.

\section{REFERENCES}

1. A. Br $\phi$ nsted and R. T. Rockafellar, On the subdifferentiability of convex functions, Proc. Amer. Math. Soc. 16 (1965), 605-611.

2. F. E. Browder, Existence and approximation of solutions of nonlinear variational inequalities, Proc. Nat. Acad. Sci. U.S.A. 56 (1966), 1080-1086.

3. - On the unification of the calculus of variations and the theory of monotone nonlinear operators in Banach spaces, Proc. Nat. Acad. Sci. U.S.A. 56 (1966), 419-425.

4. L. Danzer, B. Grünbaum and V. Klee, "Helly's theorem and its relatives," in Convexity, Proc. Sympos. Pure Math., Vol. 7, Amer. Math. Soc., Providence, R. I., 1964. 
5. C. L. Dolph and G. J. Minty, "On nonlinear integral equations of Hammerstein type," in Nonlinear Integral Equations, P. Anselone (editor), Univ. of Wisconsin Press, Madison, Wis., 1964.

6. B. Grünbaum, A generalization of theorems of Kirszbraun and Minty, Proc. Amer. Math. Soc. 13 (1962), 812-814.

7. C. Lescarret, Cas d'addition des applications monotones maximales dans un espace de Hilbert, C. R. Acad. Sci. Paris 261 (1965), 1160-1163.

8. J.L. Lions and G. Stampacchia, Inéquations variationnelles non coercives, C. R. Acad. Sci. Paris 261 (1965), 25-27.

9. - Variational inequalities (to appear)

10. G. J. Minty, On the simultaneous solution of a certain system of linear inequalities, Proc. Amer. Math. Soc. 13 (1962), 11-12.

11. - On a 'monotonicity' method for the solution of nonlinear equations in Banach spaces, Proc. Nat. Acad. Sci. U.S.A. 50 (1963), 1038-1041.

12. - On the solvability of nonlinear functional equations of 'monotonic' type, Pacific J. Math. 14 (1964), 249-255.

13. - A theorem on maximal monotonic sets in Hilbert space, J. Math. Anal. Appl. 11 (1965), 434-439.

14. R. T. Rockafellar, Characterization of the subdifferentials of convex functions, Pacific J. Math. 17 (1966), 497-510.

15. G. Stampacchia, Formes bilinéaires coercitives sur les ensembles convexes, C. R. Acad. Sci. Paris 258 (1964), 4413-4416.

16. P. Hartman and G. Stampacchia, On some non-linear differential-functional equations, Acta Math. 115 (1966), 271-310.

17. F. E. Browder, Existence and perturbation theorems for nonlinear maximal monotone operators in Banach spaces, Bull. Amer. Math. Soc. 73 (1967), 322-327.

18. - Nonlinear maximal montone operators in Banach spaces, (to appear).

INDIANA UNIVERSITY AND

TOKYO UNIVERSITY 